\title{
From Intermodulation Components to Perception and Cognition- a review
}

\author{
Noam Gordon $^{1 *}$, Jakob Hohwy ${ }^{1}$, Matthew James Davidson ${ }^{2,3}$, Jeroen van Boxtel ${ }^{2,3,4}$, Naotsugu Tsuchiya ${ }^{2,3}$ \\ 1. Cognition and Philosophy Lab, Philosophy Department, Monash University, Clayton VIC 3800, Australia \\ 2. Monash Institute of Cognitive and Clinical Neurosciences, Monash University, Clayton VIC 3800, Australia \\ 3. School of Psychological Sciences, Monash University, Clayton VIC 3800, Australia \\ 4. School of Psychology, Faculty of Health, University of Canberra, Canberra, Australia \\ *Correspondence: Noam.Gordon@monash.edu
}

\begin{abstract}
Perception results from complex interactions among sensory and cognitive processes across hierarchical levels in the brain. Intermodulation (IM) components, used in frequency tagging neuroimaging designs, have emerged as a promising direct measure of such neural interactions. IMs have initially been used in electroencephalography (EEG) to investigate low-level visual processing. In a more recent trend, IMs in EEG and other neuroimaging methods are being used to shed light on mechanisms of mid- and high-level perceptual processes, including the involvement of cognitive functions such as attention and expectation.

Here, we provide an account of various mechanisms that may give rise to IMs in neuroimaging data, and what these IMs may look like. We discuss methodologies that can be implemented for different uses of IMs and we demonstrate how IMs can provide insights into the existence, the degree and the type of neural integration mechanisms at hand. We then review a range of recent studies exploiting IMs in perception research, placing an emphasis on high-level visual processes. We conclude by suggesting future directions that can enhance the benefits of IM-methodology in perception research.
\end{abstract}

Keywords: Intermodulation (IM) components, frequency tagging, perception, signal integration, neural interactions, EEG, MEG

Abbreviations: $\underline{\mathrm{HFT}}-\mathrm{Hierarchical}$ frequency tagging. IM- Intermodulation. MSPC- Multi-spectral phase coherence. MSPCres- Multi-spectral phase coherence (response). MSPCstim- Multi-spectral phase coherence (stimulus). SSVEP-Steady-state visual evoked potential. SWIFT- Semantic waveletinduced frequency tagging.

\section{Highlights}

Intermodulation (IM) components provide a measure of signal interaction in perception

IMs can provide insights about the existence, degree and type of signal interactions

We synthesize recent developments in IM research and methods

We provide clear suggestions for analysis and interpretation of IM data 


\section{Introduction}

The elaborate and rich perceptions we experience every moment are the result of complex interactions between diverse sensory and cognitive processes across multiple hierarchical levels in the brain. Obtaining a direct physiological measure for such neural interactions is a long-term aspiration for the study of perception. This review focuses on a suggested direct measure of (nonlinear) signal interactions, namely the intermodulation (IM) frequency components associated with frequency tagging methodologies.

IM studies are based on the use of frequency tagging methods in which stimuli are modulated at a given frequency over time. As activity at the neural populations processing these stimuli becomes synchronized with the stimuli, the evoked response in the brain will exhibit the same periodicities that exist in the stimuli. This 'neural entrainment' specificity will remain for the duration that stimuli are being processed, resulting in a so-called steady-state response. In the well-known visual frequency tagging method, the steady-state visual evoked potential (SSVEP) is typically elicited by modulating the stimuli's contrast or luminance at given frequency. Frequency-domain analyses of the recorded neural response then allow matching frequency-specific brain activity to the processing of the modulated stimulus. A peak in the signal's power spectrum at the stimulus modulation frequency provides a marker or 'tag' for the stimulus-specific neural response (for a review see (Norcia et al., 2015)). (Such methods should not to be confused with various frequency-band analyses that examine spontaneous neural oscillations occurring at typical frequency ranges which may not be present in the stimulus itself.)

In more advanced designs, different elements of the stimulus can be modulated using more than one frequency (e.g., F1 and F2; note that throughout this paper we use uppercase letters to denote input/stimulus frequencies and lowercase letters to denote output/response frequencies). Importantly, not only does this allow one to separate and match specific elements of the recorded neural responses to well-defined elements of the stimulus, it can provide an additional measure for the interaction between those signals in the form of IM frequency components (also known by other names, such as IM terms, IM products, IM distortion, cross-modulation components). In such experimental designs, peaks in the power spectrum of the signal may appear at the fundamental modulation frequencies (e.g. $\mathrm{f} 1$ and $\mathrm{f} 2$ ), their harmonics, which are integer multiples of the fundamental frequencies (e.g. $2 f 1,3 f 2$, etc.) and their IMs, which appear as linear combinations of the input frequencies (e.g. 2f1+f2, f2-f1, etc.) (Figures 1 and 2). Crucially, such IMs appear as a result of, and provide direct evidence for, the non-linear integration of the neural signals driven by the differentially modulated stimulus elements (Regan and Regan, 1988b). 


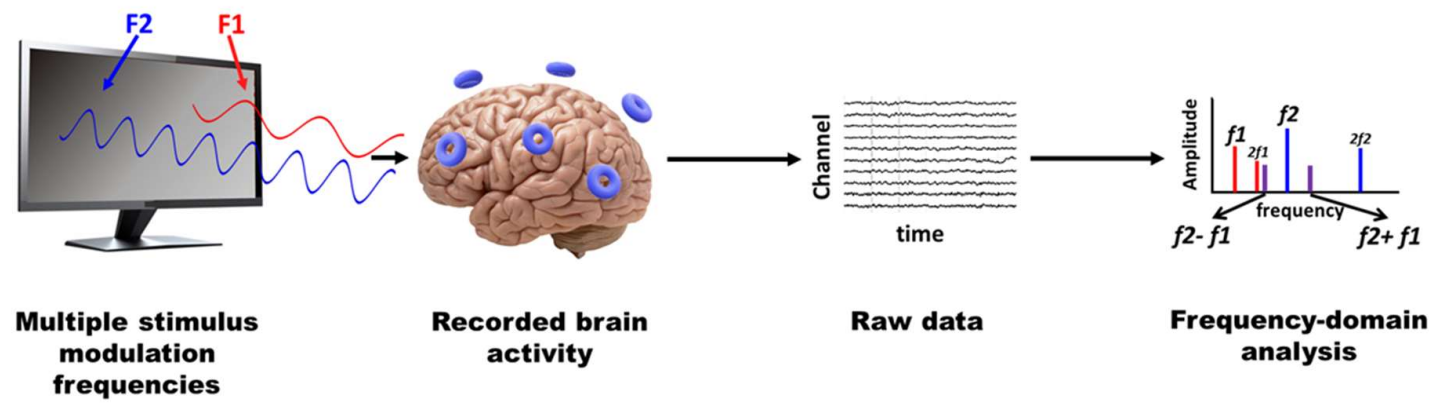

Figure 1- Basic principles of intermodulation components in perception research

Intermodulation studies are based on the use of two (or more) temporal modulations of a stimulus. Different elements of the stimulus are modulated using distinct frequencies. Brain activity is recorded during stimulus presentation and is later analysed in the frequency-domain. Neural responses entrained by the stimulus can yield peaks in power at the frequencies at which the stimulus was modulated. Given the myriad non-linear neural processes occurring in the brain, peaks are likely to be found also at various harmonics (integer multiples) of the fundamental modulation frequencies. Any non-linear integration of the signals that are driven by the unique modulation frequencies may result in peaks at additional frequencies - the intermodulation (IM) components (i.e. any linear combination of the fundamental frequencies). Such IMs do not arise when the signals do not interact (e.g. (Fuchs et al., 2008)). As such, IMs provide direct neurophysiological evidence for the non-linear integration of the different neural signals.

IM components have been demonstrated in EEG data as early as 1982 when Ratliff and Zemon reported preliminary experiments in which the contrast of adjacent segments of a windmilldartboard pattern was modulated using two different frequencies. They observed that these patterns evoked power at some intermodulation frequencies and concluded they result from lateralinteractions along the visual processing pathway (Zemon and Ratliff, 1982).

IMs are being used in recent years to shed light not only on low-level visual processes but also on mechanisms of mid- and high-level perception (e.g. (Boremanse et al., 2013; Giani et al., 2012)), including the involvement of cognitive functions such as attention and expectation (e.g. (Gordon et al., 2017; Kim et al., 2017)). IMs have also recently been extended to Brain Computer Interface studies (Chen et al., 2013), underscoring the potential of IMs in the neurosciences.

Utilizing IMs for the study of mid- or high-level perception requires the use of more advanced experimental designs and analyses. In terms of experimental stimuli, for example, various visual illusions can be tailored to elicit IM components (Aissani et al., 2011; Alp et al., 2016; Gundlach and Müller, 2013). Other studies have combined contrast-modulation SSVEP methods with more advanced image-content based frequency tagging (e.g. Semantic Wavelet-Induced Frequency Tagging (SWIFT; (Koenig-Robert and VanRullen, 2013)) to induce IMs across multiple hierarchical levels (Gordon et al., 2017).

However, in spite of the increasing prominence and potential of IMs in neuroscience, the literature remains scattered and experimental methodologies are largely exploratory. The goal of this review is to provide an understanding of the various mechanisms that may give rise to IMs, organise the knowledge one can obtain from IMs and discuss methodologies that can be implemented for various experimental purposes. The review focuses on the use of IMs in electroencephalography (EEG) and magnetoencephalography (MEG) studies, placing a particular emphasis on the benefit of IMs in the study of high-level visual processes. 


\subsection{Definitions}

IM components are linear combinations of the input frequencies that result from the non-linear integration of multiple input frequencies. This definition can be unpacked as follows:

Non-linear integration of multiple input frequencies- A system or function is considered non-linear if the combination of two inputs does not necessarily yield the sum of their respective outputs (i.e. when the additivity property $f(x+y)=f(x)+f(y)$ is not satisfied for all $x$ and $y$ values. (Note that for simplicity this review does not consider the other requirement of linearity in mathematics, namely the homogeneity property: $f(a x)=a f(x)$ for all a). A system being non-linear implies that its behaviour cannot be described just in terms of its constituent parts. Rather, its overall function (output) depends also on interactions which may vary across different input values.

The mathematician Stanislaw Ulman said that "Using a term like non-linear science is like referring to the bulk of zoology as the study of non-elephants" (Campbell et al., 1985). This conveys the notion that the vast majority of natural systems are, in reality, non-linear. This includes cortical function. To begin with, synaptic transmissions - the primary neuronal communication method - are to a large extent non-linear (Markram, 2003). Spike-thresholding, rectification and saturating transduction are all examples for non-linear stimulus-response relationships at the single-neuron level. It has been suggested that non-linear behaviors of local neural activity can generate approximately linear behaviors at the more macroscopic levels (Boynton et al., 1996; Shapley, 2009). Nevertheless, numerous non-linear neural processes, as well as psychophysical outcomes, have been described in the literature. Pooling neuronal responses across multiple units can allow non-linear normalization and gain control mechanisms (Carandini and Heeger, 2011). Indeed, non-linear neuronal responses have been repeatedly demonstrated across different areas in the cortex (e.g. sigmoid-like response profiles, phase invariance in complex V1 cells (Zhu and Rozell, 2013)) and various neuronal circuitries for implementing non-linear neuronal operations have been suggested (Kouh and Poggio, 2008).

Non-linear integration of multiple input frequencies- the terms integration and interaction are often used in relation to IMs. In the context of frequency analyses, linear interactions may refer, for example, to neural synchronization at the same frequency (e.g. stimulus F1 $\rightarrow$ output f1) whereas non-linear interactions can refer to synchronization between multiple frequencies such as harmonics and IMs. Throughout this paper we will therefore refer either to the non-linear integration of, or to the interaction between neural signals (avoiding the use of 'non-linear interactions'). Non-linear processes can of course involve single-frequency inputs as well, in which case the recorded response signal may include harmonics of the fundamental modulation frequency regardless of any interactions. This should be kept in mind since, as we discuss later, extracting the greatest interpretive value from the IMs requires considering them in conjunction with the fundamental and harmonic frequencies.

\subsection{Examples of intermodulations in simple simulations}

To illustrate possible input-output relationships, and conditions under which harmonics and IMs appear in the output, Figure 2 shows different mathematical functions and their respective frequency decompositions. The inputs of most functions were based on two sinusoids of different frequencies $(1.3 \mathrm{~Hz}$ and $10 \mathrm{~Hz})$ with additional uncorrelated random Gaussian noise: $\mathrm{X}=$ $\sin \left(2 \pi^{*} 1.3 \mathrm{t}\right)+\varepsilon_{\mathrm{t}} 1$ and $Y=\sin (2 \pi * 10 \mathrm{t})+\varepsilon_{\mathrm{t}} 2$ ( $\varepsilon$ represents independent and identically distributed noise 
terms added to better approximate neural responses which are not perfectly sinusoidal and to demonstrate the relative robustness of IMs to such noise). In addition to sinusoids, we used a rectification function ( $\operatorname{Rec}(x)=x$ if $x>0$ and 0 otherwise) as well as a square wave $(S q(x)$ is defined as 1 if $\sin (x)>0$ and -1 otherwise).

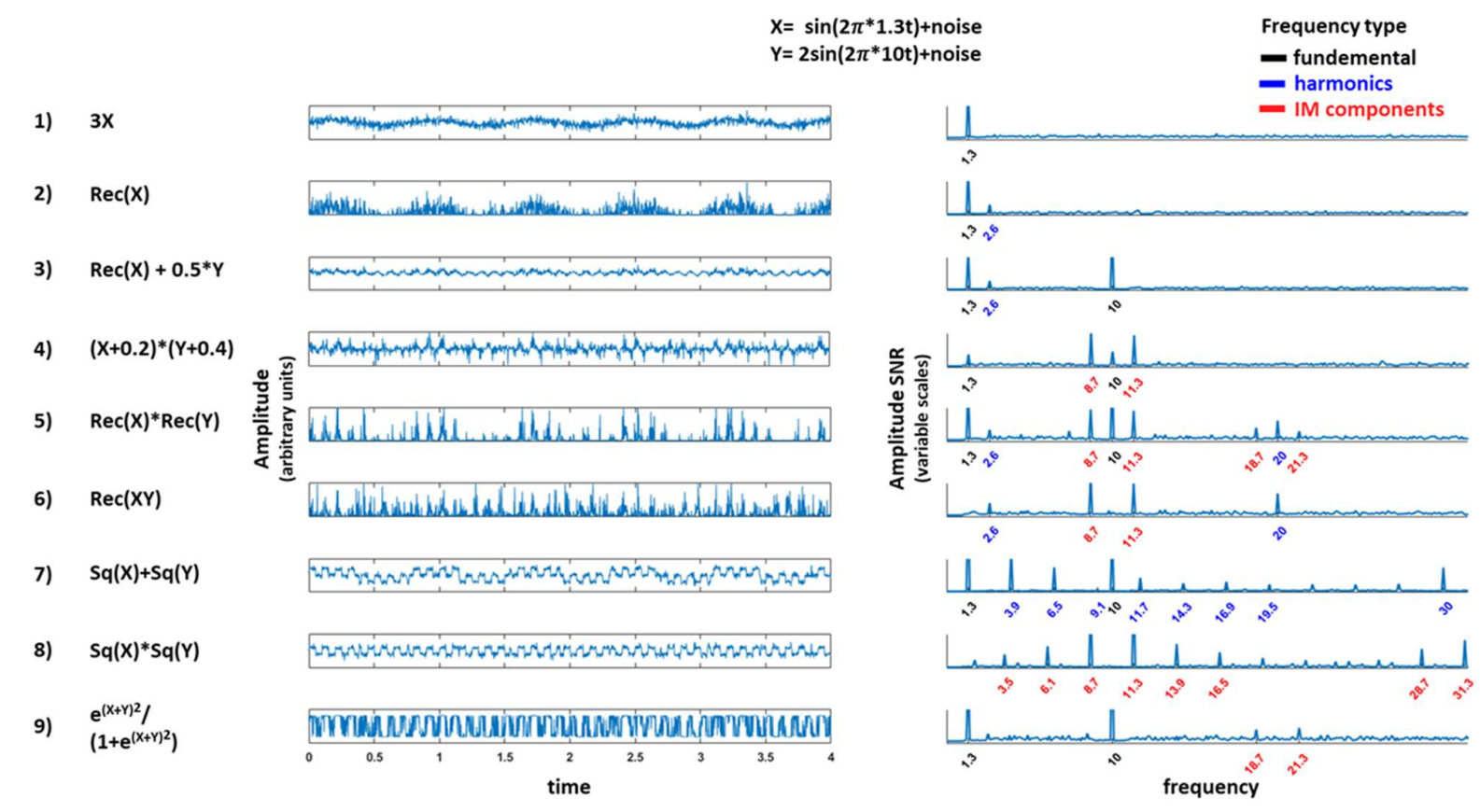

Figure 2- Examples of different mathematical functions and their respective spectra

Different mathematical functions and their respective frequency decompositions. Peaks at the fundamental frequencies are marked with black numbers, at harmonic frequencies with blue numbers and at IM components with red numbers. The term 'fundamental' frequencies denotes the frequencies of the input signal ( $1.3 \mathrm{~Hz}$ and $10 \mathrm{~Hz}$ here), the term 'harmonic' denotes any integer multiple of the fundamental (e.g. the $3 \mathrm{rd}$ harmonic of $\mathrm{F}=1.3 \mathrm{~Hz}$ is $3 * 1.3 \mathrm{~Hz}=3.9 \mathrm{~Hz}$ ). The fundamental frequency is, by this definition, the 1st harmonic), and the term intermodulation (IM) denotes, any linear combination of the fundamental frequency (i.e. $n f 1+m f 2 ; n, m=+-1,+-2,+-3 \ldots$.). The IM 'order' refers to the sum of the absolute values of the integer coefficients defining the IM component (e.g. $13.9 \mathrm{~Hz}$ here is considered a 4th order IM since $13.9 \mathrm{~Hz}=3 * 1.3+1 * 10$ $\mathrm{Hz}$ and $3+1=4$, and $8.7 \mathrm{~Hz}$ is considered 2 nd order as $8.7=(-1) * 1.3+1 * 10$ and abs $(-1)+1=2$.). Table 1 below describes the different functions used here, their potential relevance to neural processes, and the dominant frequencies in their output spectra.

Table 1.

\begin{tabular}{|l|l|l|l|l|l|}
\hline & Function & \multicolumn{1}{|c|}{ Description } & \multicolumn{1}{|c|}{$\begin{array}{c}\text { Potential neural } \\
\text { relevance }\end{array}$} & \multicolumn{1}{|c|}{ Output spectrum } & \multicolumn{1}{c|}{ Comment } \\
\hline 1 & $3 \mathrm{X}$ & $\begin{array}{l}\text { A single input } \\
\text { frequency processed } \\
\text { linearly }\end{array}$ & $\begin{array}{l}\text { Only the fundamental } \\
\text { frequency. }\end{array}$ & $\begin{array}{l}\text { Linear functions don't } \\
\text { yield harmonics or IMs. }\end{array}$ \\
\hline 2 & $\operatorname{Rec}(\mathrm{X})$ & $\begin{array}{l}\text { Non-linear processing } \\
\text { of a single input. A half } \\
\text { rectifier (negative } \\
\text { values replaced with } \\
\text { 0). }\end{array}$ & $\begin{array}{l}\text { Inhibitory influences } \\
\text { can bring firing rates } \\
\text { down to 0 but not } \\
\text { below }\end{array}$ & $\begin{array}{l}\text { The fundamental and } \\
\text { harmonics (in this case } \\
\text { only the 2 }\end{array}$ & $\begin{array}{l}\text { Non-linear processing } \\
\text { yields harmonics. }\end{array}$ \\
\hline
\end{tabular}




\begin{tabular}{|c|c|c|c|c|c|}
\hline 3 & $\begin{array}{l}\operatorname{Rec}(\mathrm{X}) \\
+ \\
0.5^{*} \mathrm{Y}\end{array}$ & $\begin{array}{l}\text { One input processed } \\
\text { non-linearly (half } \\
\text { rectified) and the other } \\
\text { linearly. Input signals } \\
\text { do not interact. }\end{array}$ & $\begin{array}{l}\text { Parallel processing of } \\
\text { different neural } \\
\text { signals }\end{array}$ & $\begin{array}{l}\text { The fundamental and } \\
2^{\text {nd }} \text { harmonic of the } \\
\text { first input term and } \\
\text { the fundamental of } \\
\text { the second input term. }\end{array}$ & $\begin{array}{l}\text { Non-linearity results in } \\
\text { harmonics, but pure } \\
\text { linear summation of } \\
\text { signals (without } \\
\text { interaction) does not } \\
\text { yield IMs. }\end{array}$ \\
\hline 4 & $\begin{array}{c}(\mathrm{X}+0.2) \\
\quad * \\
(\mathrm{Y}+0.4)\end{array}$ & $\begin{array}{l}\text { The two inputs interact } \\
\text { via multiplication }\end{array}$ & $\begin{array}{l}\text { Multiplicative } \\
\text { operations have been } \\
\text { demonstrated in } \\
\text { various sensory } \\
\text { systems (e.g. } \\
\text { (Gabbiani et al., 2004; } \\
\text { Pena and Konishi, } \\
\text { 2004)) }\end{array}$ & $\begin{array}{l}\text { Strong second order } \\
\text { IMs. }\end{array}$ & $\begin{array}{l}\text { Under certain } \\
\text { interaction conditions } \\
\text { the IMs can be stronger } \\
\text { than the fundamentals. }\end{array}$ \\
\hline 5 & $\begin{array}{l}\operatorname{Rec}(\mathrm{X}) \\
\quad * \operatorname{Rec}(\mathrm{Y})\end{array}$ & $\begin{array}{l}\text { The two inputs are first } \\
\text { half-rectified and then } \\
\text { interact via a } \\
\text { multiplication term }\end{array}$ & \multirow[t]{2}{*}{$\begin{array}{l}\text { A sequence of neural } \\
\text { computations }\end{array}$} & $\begin{array}{l}\text { Fundamentals, } \\
\text { harmonics, } 2^{\text {nd }} \text { and } 3^{\text {rd }} \\
\text { order IMs }\end{array}$ & \multirow{2}{*}{$\begin{array}{l}\text { In addition to the types } \\
\text { of non-linear operations } \\
\text { See } 2-4 \text { ), the spectra } \\
\text { depends on the order at } \\
\text { which they are carried } \\
\text { out (compare } 5 \text { and } 6 \text { ). }\end{array}$} \\
\hline 6 & $\operatorname{Rec}(X Y)$ & $\begin{array}{l}\text { The two inputs first } \\
\text { interact via a } \\
\text { multiplication term and } \\
\text { are then half rectified } \\
\text { together. }\end{array}$ & & $\begin{array}{l}\text { Harmonics and IMs, } \\
\text { but notably different } \\
\text { than the previous } \\
\text { example, e.g. the } \\
\text { absence of } \\
\text { fundamentals }\end{array}$ & \\
\hline 7 & $\begin{array}{l}\text { Square } \\
\text { wave } 1 \\
\quad+ \\
\text { Square } \\
\text { wave } 2\end{array}$ & $\begin{array}{l}\text { Sum of two square } \\
\text { waves. }\end{array}$ & $\begin{array}{l}\text { Sustained input from } \\
\text { ON/OFF cells }\end{array}$ & $\begin{array}{l}\text { Many odd-order } \\
\text { harmonics }\left(1^{\text {st }}, 3^{\text {rd }}, 5^{\text {th }} \text {, }\right. \\
\text { etc. })\end{array}$ & $\begin{array}{l}\text { The Fourier } \\
\text { decomposition of non- } \\
\text { sinusoidal signals } \\
\text { contains power at } \\
\text { harmonic frequencies. } \\
\text { Square waves yield } \\
\text { many odd-order } \\
\text { harmonics. }\end{array}$ \\
\hline 8 & $\begin{array}{l}\text { Square } \\
\text { wave } 1 \\
* \\
\text { Square } \\
\text { wave } 2\end{array}$ & $\begin{array}{l}\text { Multiplication of two } \\
\text { square waves. }\end{array}$ & $\begin{array}{l}\text { Coincidence detection } \\
\text { of ON/OFF cells }\end{array}$ & $\begin{array}{l}\text { Many low and high- } \\
\text { order IMs including all } \\
\text { combinations of the } \\
\text { harmonics seen in the } \\
\text { previous function. }\end{array}$ & $\begin{array}{l}\text { Interactions between } \\
\text { non-sinusoidal waves } \\
\text { can result in many high- } \\
\text { order IMs. }\end{array}$ \\
\hline 9 & $\begin{array}{c}\mathrm{e}^{(X+Y)} / \\
\left(\mathrm{e}^{(X+Y)}+1\right)\end{array}$ & $\begin{array}{l}\text { A sigmoid function with } \\
\text { the exponent being the } \\
\text { sum of the two inputs }\end{array}$ & $\begin{array}{l}\text { Firing rates often } \\
\text { modelled as sigmoids }\end{array}$ & $\begin{array}{l}\text { Fundamentals and 3rd } \\
\text { order IMs }\end{array}$ & \\
\hline
\end{tabular}

As demonstrated in Figure 2, the emergence of IMs occur uniquely from the interaction between input signals, and the output spectra depends on the specific integration mechanisms at play. The functions used in this figure are arguably much simpler than real neural processes, which result from complex and multi-layered interactions. Nevertheless, they illustrate why, as further discussed in section 3, the output spectrum of steady-state responses may provide insight into the existence, degree and type of neural interactions at hand. These interactions have been evoked by a variety of methodologies in the empirical literature, which we now review. 


\section{Methodologies}

The majority of IM studies have been performed using EEG, though we discuss results from some studies that used MEG and ElectroCorticoGraphy (ECOG) as well. All these methods enjoy a higher temporal precision compared to other neuroimaging methods (such as $\mathrm{FMRI}$ and PET) and provide a direct measure of population-wide neural activity (Cohen, 2017). As such, they are particularly suitable for the examination of precisely-defined individual frequency components in the context of perception and cognitive function.

A wide range of stimulus parameters and analysis methods have been used across different IM studies. While it is difficult to a priori determine the optimal methods for each purpose, it is important to be aware of the notable impact these choices may have on the results. In this section, we discuss some of the essential differences between stimulus parameters and analysis methods used across studies.

\subsection{Stimulus parameters and analysis methods}

An important choice is which modulation frequencies to use. Various modulation frequencies have been applied, typically ranging from $1 \mathrm{~Hz}$ to $20 \mathrm{~Hz}$. The use of different frequencies can impact various aspects of a study since neural mechanisms may be tuned differently to specific input frequencies (Vialatte et al., 2010). Indeed, different cortical networks have been shown to be maximally tagged by different flicker frequencies (Ding et al., 2006), a phenomenon known as 'resonance frequency' (Herrmann, 2001). In addition, both sinusoidal (e.g. (Boremanse et al., 2014; Tsai et al., 2012)) and square (e.g. (Gundlach and Müller, 2013; Hou et al., 2003)) waveforms have been applied at various modulation depths. As demonstrated in Figure 2, using square or sinusoidal waves can result in significantly different output spectra. This should be kept in mind when comparing results across different studies.

Spectral responses at different frequencies may stand out more or less depending on those of their neighborhood frequencies (e.g., spontaneous alpha waves $(8-12 \mathrm{~Hz})$ or common muscular artifacts (MEG; 20-300 Hz (Muthukumaraswamy, 2013)). Influences of the neighborhood frequencies can be mitigated by calculating the signal-to-noise ratio (SNR) of the spectral output, by which the power at each frequency is compared to the power at closely spaced neighbourhood frequencies. A common method for calculating SNRs is to divide the power (or amplitude) at a given frequency by the mean power (or amplitude) of several adjacent frequency bins from each side (if the geometric mean is used, rather than the arithmetic mean, log of the SNR is equivalent to subtracting the arithmetic mean of the logarithm of the power across nearby frequencies from that at a given frequency). Non-specific artifact or background activity typically evoke a broadband spectral response, thus the SNR between neighbouring frequencies is typically low. Conversely, the defining frequencies of steady-state responses are narrow-band, thus their SNR in the frequency domain can be high.

The Fourier half-bandwidth ( $\Delta \mathrm{f}$ : the frequency resolution which signifies the ability to separate frequency components of a Fourier transform) is inversely determined by the duration of the stimulus ( $\Delta \mathrm{f}=1$ /duration(sec) for a single taper Fourier transform (Mitra and Pesaran, 1999)). Using longer stimulus durations can therefore increase narrow-band SNRs and significantly improve the ability to separate stimulus-specific neural responses from non-specific activity (Srinivasan et al., 
2006). It is therefore important to select modulation frequencies and epoch durations that yield a fourier half-bandwidth small enough to distinguish the frequencies-of-interest (fundamentals, harmonics, and IMs) from one another in subsequent analysis.

Some theoretical considerations may bias the choice of modulation frequencies toward specific ranges. These may include, for example, the resonance characteristic of the region or modality of interest (Gulbinaite et al., 2017; Herrmann, 2001; Lea-Carnall et al., 2016). It is nevertheless difficult to determine a priori whether one set of parameters would be superior to another for any given application. This is partly due to individual differences in the efficacy of SSVEP entrainment, as the strength of spectral output can be determined by endogenous brain oscillations (Gulbinaite et al., 2017). Each parameter combination may also allow specific aspects of neural processes to be revealed more so than others, depending on the type of non-linear computations that can be implemented by the neural mechanism at hand, the specific non-lineraties that can be induced by the experimental stimuli, and the ability of the imaging method (EEG, ECoG, etc.) to reveal them.

Choosing parameters therefore remains, to a considerable extent, an empirical and study-specific matter. Therefore, direct comparisons between studies are not trivial and require considering the specific measures and parameters used for both stimulus preparation and data analysis.

\subsection{Amplitude vs Phase analysis}

Distinct aspects of non-linear interactions may be revealed by examining both phase and amplitude of the spectrum. Most IM studies used derivatives of the Fourier transform amplitude at each frequency component to quantify IM strength. This includes the amplitude (e.g. (Gundlach and Müller, 2013; Tsai et al., 2012)), its squared value (i.e. power ; e.g. (Katyal et al., 2016)) and their respective SNRs (e.g. (Boremanse et al., 2014; Cunningham et al., 2017; Kim et al., 2017)). Conversely, phase analyses have only recently been applied in IM studies.

The reason that only few studies have implemented phase analyses for IMs might be that traditional phase-synchrony measures (Palva and Palva, 2012) are less suitable for studying multiple IMs. The consistency of a single frequency phase is characterized in two ways. The first popular measure focuses on a single channel and analyzes the inter-trial phase consistency at any given frequency with respect to exogenously triggered repeated events. The second one instead focuses on the phase relationship between channels, which can characterize phase consistency over time with or without aligning the responses with respect to exogenous events. While IM research can benefit from these two approaches, the more interesting and promising measure would be the one that examines relationship among the phases of any given IM component and the driving (fundamental) frequencies. Phase-synchrony measures, such as the bi-phase locking value (bPLV) have been proposed to quantify the phase-coupling between two fundamental frequencies and their IM frequency, however, these methods are limited only to the 2 nd order sum IM (f1+f2).

Recently, Yang et al introduced a novel phase-coupling measure for quantifying any IM component (Yang et al., 2016). This phase measure - the multi-spectral phase coherence (MSPC) - is especially useful for the study of IMs since it quantifies the consistency of the phase relationships among an IM component and the fundamental frequencies within a single channel (Figure $3 \mathrm{~A}$ and $3 \mathrm{~B}$, and see additional details in section 3.3). In section 3.3 we discuss recent developments introduced by Gordon et.al (Gordon et al., 2018) that expand the use of MSPC analyses in the context of hierarchical perceptual processing. 
A

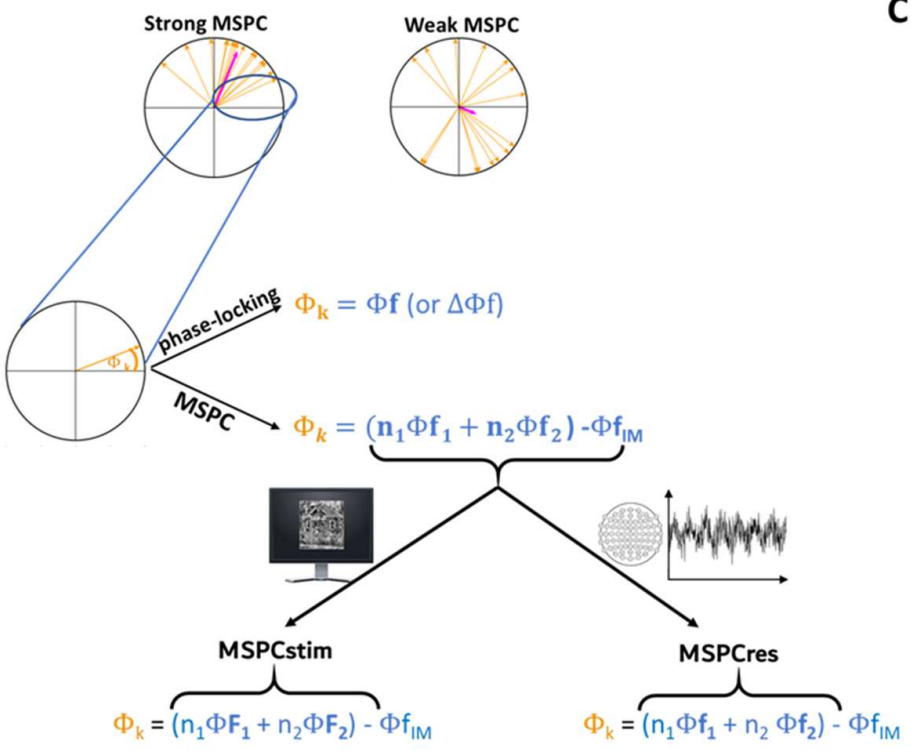

C

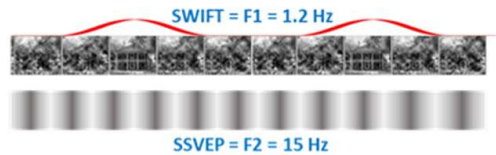

SSVEP $=\mathrm{F} 2=15 \mathrm{~Hz}$
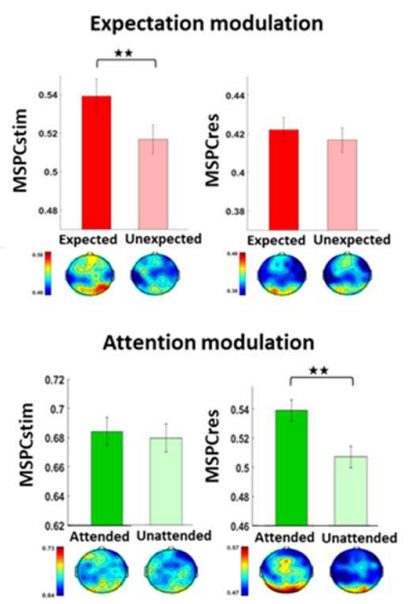

Figure 3- Multi-spectral phase coherence (MSPC)

The MSPC measure quantifying IM components. MSPC quantifies the consistency of the phase relationships among the driving fundamental frequencies and the the IM across trials within a single channel. (A) Examples of stronger (left) and weeker (right) MSPC values. The angle of each orange vector in the unit circle is calculated from a single trial/epoch and represents the relationship between the phase of the IM component and the phases of the fundamental frequencies within that trial. The greater the consistency e) of the difference phase angles across trials, the greater the mean vector length (pink vector). (B) Comparison between the classical phase-locking-value (PLV) and the MSPC. For the PLV, the phase term within each trial is based on a single frequency or frequency band (either within a single channel or the inter-channel phase difference). For MSPC, the phase term is based on the difference $(\Delta \phi)$ between the phase of the IM component $\left(\phi f_{I M}\right)$ and the (weighted) sum of the phases of the fundamental frequencies $\left(n_{1} \phi f_{1}+n_{2} \phi f_{2}\right)$ within each channel and each epoch. This way, the MSPC aims to quantify the degree to which the phase of the IM is driven by the phases of the fundamental frequencies. Gordon et al. (Gordon et al., 2018) further distinguished between what they termed 'MSPCstim' and 'MSPCres' which use either the stimulus phases (the on-screen image, bottom left, upper-case F1 and F2), or the EEG response phases (right, lower case $\mathrm{f} 1$ and $\mathrm{f} 2$ ) as the driving fundamental frequencies. The weights of the fundamental frequencies, $\mathrm{n} 1$ and $\mathrm{n} 2$, are the coefficients that define the IM frequency (e.g., given $\mathrm{F} 1=1.2 \mathrm{~Hz}$ and $\mathrm{F} 2=15 \mathrm{~Hz}$, the weights for the 3 rd order IM component $2^{*} F 1+F 2=17.4 \mathrm{~Hz}$ would be $\mathrm{n} 1=2$ and $\mathrm{n} 2=1$ ). (C) A functional dissociation between MSPCstim and MSPCres. Gordon et al. examined how the integration of top-down and bottom-up signals is modulated by expectation and attention (Gordon et al., 2018). To do so, they used the Hierarchical Frequency Tagging (HFT) method in which two frequency tagging methods are combined - a global contrast modulation to induce steady-state visual evoked potentials (SSVEP) and a semantic wavelet-induced frequency tagging (SWIFT) which tags category-specific activity at higher visual levels. Using the MSPC measure, the authors showed that both expectation and attention increase the degree of integration between top-down and bottom-up information, albeit via distinct pathways as reflected by the different modulations of the MSPCstim and MSPCres measures.

The interaction between distinct frequency bands have also been characterized using the phaseamplitude cross-frequency coupling (CFC) measure which quantifies the dependence between the phase of a low-frequency band and the amplitude (or power) of a high-frequency component (Canolty and Knight, 2010). To the best of our knowledge the CFC measure has not been applied to IMs, though it is possible that such CFC methods can be generalized for use with multiple IM components in much the same way as the MSPC measure generalized the phase-synchrony approach for this purpose. 


\section{What we can learn from IMs}

IM analyses can provide insights into the existence, the degree and the type of the neural interactions at hand. Here, we discuss the methodologies required to inform each of these aspects. Notably, they require an increasing level of sophistication, arguably accompanied by a decreased ability to draw unequivocal and decisive conclusions.

3.1 The existence of neural interactions. On the most basic level, finding statistically significant IM components in the recorded output provides a direct and definite indication for the existence of interactions between distinct neural signals. To understand IM's directness and definiteness, consider popular alternative methods in neuroimaging. For example, multisensory integration has been classically identified using conjunction or interaction analyses in fMRI. In conjunction analyses, multisensory integration is inferred if a single region responds to inputs from two (or more) sensory modalities. However, one limitation of the conjunction analyses is that it cannot detect interactions in which the signal from one sensory modality modulates the response elicited by the other modality, despite no significant response being elicited by the first modality when presented alone. In interaction analyses, interactions are inferred by examining whether the response to a multisensory stimulus differs from the sum of the responses to each of the unisensory stimuli (Calvert et al., 2001; James and Stevenson, 2012). However, there are several downsides to this approach as well. For example, ambiguities may be introduced by nonlinearities or ceiling effects in the BOLD, rather than the neuronal response (Noppeney, 2012). In comparison, the presence of the IMs does not pose these problems as it definitively shows direct evidence of the presence of nonlinear integration mechanisms.

3.2 The degree of signal integration. Comparing between IMs obtained in different, yet carefully controlled conditions, may shed additional light on the degree of non-linear integration underlying different perceptual or cognitive processes. For example, one can try to determine if the neural signals driven by the different stimulus modulations are integrated more efficiently under one experimental condition than another. The ability to link IM modulations to condition-dependent changes in non-linear integration nevertheless requires analysing the IMs also in relation to the fundamental and harmonic frequencies. For example, consider a case where the power at the IMs is proportional to the power at the fundamental and harmonic frequencies. This finding suggests that $100 \%$ of the variation in IM power can be accounted for by variations in the power at the fundamental and harmonic frequencies. In such a case, changes in IM power are unlikely to reflect modulations of the actual integration processes. Conversely, Figure 4 shows examples from two different studies where IMs increased as a function of the experimental condition while the power at the fundamental and/or the harmonics in fact decreased. Effects such as these are not possible to explain in terms of a simple dependency of IMs on the fundamentals. Indeed, it is notably easier to link IMs to condition-specific variations in signal-integration when the fundamental frequencies (and their harmonics) do not exhibit the same trend as the IMs, or when they even behave in opposite ways (Figure 4; e.g. (Gordon et al., 2017; Zhang et al., 2011). Arguably, normalising the total power across a wide range of IMs by the total power across the fundamental and harmonic frequencies, within each participant, may also provide a valid measure for the dissociation of changes in IMs from changes in the fundamental frequencies (Zhang et al., 2011). 

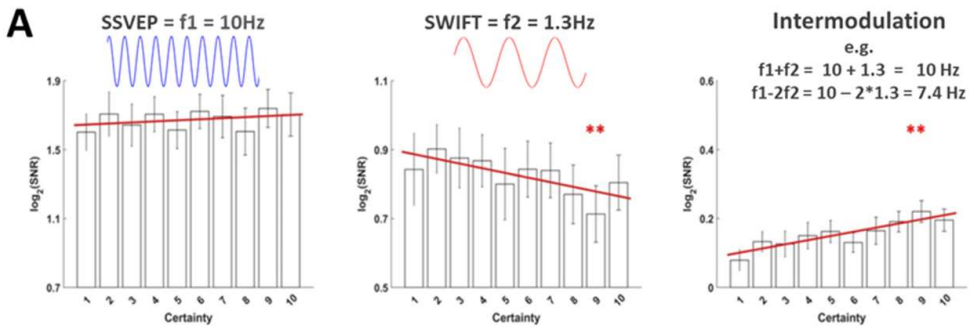

B

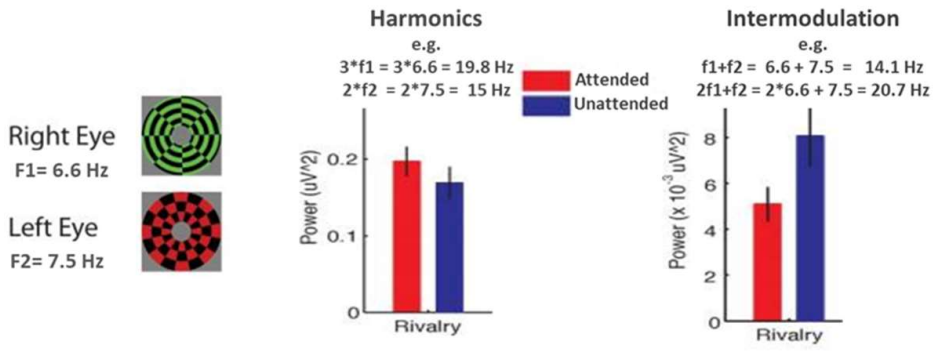

Figure 4- Dissociating responses at IMs from responses at the fundamental and harmonics

IMs needs to be interpreted in relation to the fundamentals and it is important to examine whether the changes in IMs can be accounted for by changes in the fundamental and harmonic frequencies. A) and B) are the exemplars in which the power at IMs are dissociated from the power at the fundamentals. This effect cannot be explained by a simple dependency of IMs on the fundamentals. (A) In a Hierarchical Frequency Tagging study by Gordon et al. the authors combined two frequency-tagging methods - the steady-state visual evoked potential (SSVEP) and the semantic wavelet-induced frequency tagging (SWIFT) to tag activity at lower and at higher visual levels, respectively (reproduced with permission from (Gordon et al., 2017)). They showed that the IMs increased with certainty (expectation) despite the opposite trend at the fundamental tagging frequencies and concluded that the integration of top-down and bottom-up signals increases with expectation. (B) In a study by Zhang et al. the authors presented different flickering stimuli to each eye to induce binocular rivalry (reproduced with permission from (Zhang et al., 2011)). Comparing between attended and unattended conditions, they demonstrated a significant increase in IM power during the unattended condition despite a decrease in power at the fundamental and harmonics. They concluded that the interaction between the two eyes' signals was stronger when attention was withdrawn.

To provide evidence that variations in IM responses indeed signify more than simple variations in the strength of the 'input' signals, one should demonstrate that the amplitude, the power or the SNR of these values at the IMs do not correlate strongly with those at the fundamental and harmonics, or that the IMs can better account for behavioral outcomes than the fundamental and harmonics can.

Such a need to dissociate the IM response from the response at the fundamental and harmonic frequencies may be relaxed to some degree when using the phase-based MSPC measure (see section 2.2). This is because MSPC, in itself, quantifies the consistency of the relationship between the fundamental frequencies and the IMs. In other words, a significant difference in MSPC between different conditions directly implies significant changes in the relationships among the fundamentals and IMs.

3.3 The type of non-linear integration. While IMs indicate the integration of distinct streams of neural signals, they do not readily point at the exact mechanisms (or type of non-linearity) that gave rise to them. Figure 2 illustrates how the pattern of harmonics and IMs could, in principle, reveal something about the underlying mechanisms, however, the functions in that figure are a clear 
simplification compared to real neural processes. Moreover, several processes involving different sequences of signal integration may occur in parallel, yielding various IM components in the recorded signal. Disentangling different IM components from each other and distinguishing between the contribution of different computational processes to the same IM component is a significant challenge. This is perhaps in much the same way as a signed business contract indicates there were interactions going on between two parties, but might not, on its own, provide clear insight regarding the specific back-and-forth negotiations that took place behind the scenes. For example, in a recent study by Katyal et al. the authors presented coherent and conflicting gratings separately to each eye (Katyal et al., 2018). The simultaneous presentation of conflicting monocular signals can result in binocular rivalry, a phenomenon whereby conscious perception alternates between each eye's image over time. Katyal et al. interpreted the significant f2-f1 IM component to reflect conflictdetecting binocular neurons yet the authors acknowledged that the same frequency component may be generated also by processes of integration of inputs from both eyes.

Despite this challenge, one can apply various analyses to seek better understanding of the underlying mechanisms that generated them. We discuss three approaches that have been implemented in different studies for this purpose.

\section{Experimentally testing competing options}

One way to evaluate the potential contribution of different neural mechanisms to the generation of the observed IMs is to design additional experiments that examine competing options in isolation. This approach was demonstrated nicely by Zhang et al (Zhang et al., 2011). There, the authors sought to examine the role of attention in resolving interocular competition in binocular rivalry. To do so, they presented two different checkerboard patterns (one to each eye) each flickering at a different frequency $(6.6$ and $7.5 \mathrm{~Hz})$. The authors introduced a central attention task to distinguish between attended and unattended conditions. Analyses were performed only on the six most posterior channels (this is important to keep in mind when comparing results from this and other studies). Notably, while the power of the harmonics increased with attention, the power at the IMs (five components ranging from $2^{\text {nd }}$ to $5^{\text {th }}$ order which had high power at frequencies below $22 \mathrm{~Hz}$ ) were strongest during the unattended condition. Two possible mechanisms were explored by the authors to explain this apparent enhancement of integration during the unattended condition. The first one was an abolishment of rivalry accompanied by 'fused' perceptions where IMs are generated, for example, by neurons combining local signals from both eyes in early visual areas. The second possibility was an increase in patchwise rivalry where IMs are generated, for example, at later visual areas where receptive fields are large enough to integrate responses from adjacent patches. To compare these options, the authors created and presented participants with two new sets of stimuli, each allowing for the emergence of either a fusion-like or a patchwise percept. Only the fusion-like simulation produced IM power compatible with the increased IM power during the unattended condition. Such a comparison allowed the authors to attribute greater likelihood for an underlying 'fusion' process. The authors concluded that without attention, rivalry ceases and the two eyes' signals locally combine in the visual cortex.

This demonstrates how additional controlled experiments can, at times, allow one to distinguish between different plausible interaction mechanisms that may account for the generation of the IMs in the recorded signal. 


\section{Modelling}

Physiologically-inspired computational models can be compared against the empirical spectrum to obtain stronger support for one model over competing ones.

Regan and Regan suggested such a systematic approach and demonstrated how the examination of IM responses (including high order terms) across a wide range of input amplitudes allowed to support, or reject, specific physiologically relevant computational models (Regan and Regan, 1989). To do so, they presented participants with a stimulus comprising two superimposed circular patches of light, while modulating the luminance of each patch with a different sinusoidal frequency. They then characterised how each IM component- reaching as high as $5^{\text {th }}$ order terms - changed as a function of varying input amplitudes, and compared various computational models against their empirical results. This allowed some candidate models to firmly be rejected, and others to gain varying levels of support.

Several studies implemented a similar approach to test contrast normalisation, gain-control and conjunction detection models against EEG data obtained when presenting various black and white patterns such as checkerboards, grating and plaids (e.g. (Candy et al., 2001; Cunningham et al., 2017). For example, Tsai et al. presented two checkerboard-like random noise patterns, contrastmodulated at $5.14 \mathrm{~Hz}$ and $7.2 \mathrm{~Hz}$ (Tsai et al., 2012). EEG response spectra were then compared against three models based on the hyperbolic ratio function. The hyperbolic ratio function is commonly used to model cortical responses that follow saturating transducer non-linearities involving contrast gain control mechanisms from nearby units. The basic model was expressed as $R=$ $C^{\wedge} p /\left(Z^{\wedge} q+C^{\wedge} q\right)$, where $R$ is the neural response measured as EEG power at certain frequencies, $C$ is the contrast of the stimulus, with $Z, p, q$ to be fitted with the data. They extended this model for use with two input-signals. The three models were able to predict the fundamental frequencies and their harmonics to a comparable degree, however, only one of the models was able to account for the characteristics of the $\mathrm{f} 1+\mathrm{f} 2 \mathrm{IM}$ component (specifically, a model that incorporated temporal integration in the gain pool with a duration of approx. $26 \mathrm{~ms}$.).

Cunningham, Baker and Peirce sought to relate IMs to mechanisms beyond V1 that are sensitive to compound stimuli (Cunningham et al., 2017). Pairs of sinusoidal gratings of different spatial frequencies were superimposed and contrast-modulated at different temporal frequencies, 2.3 and $3.75 \mathrm{~Hz}$. The resulting stimuli looked either like a checkerboard plaid (considered the "coherent" stimuli) or like transparently overlaid gratings (considered the "noncoherent" stimuli). A significant response at the $2 \mathrm{f} 1+2 \mathrm{f} 2 \mathrm{IM}$ component was found only for the coherent condition. The authors argued that this does not reflect simple lateral suppressive interactions (such as cross-orientation suppression) since such effects were not dependent on the coherency of the patterns. Comparing the data against different computational models (based on the same hyperbolic ratio function described above) the data was best accounted for by a two-stage model involving conjunction detection based on a logical AND operation.

By applying a similar methodology, Baker and Wade identified a 'winning' contrast response model which they argue may reflect a generic 'system-wide' signal-combination algorithm in perception (Baker and Wade, 2017). There, six models were compared against each other, differing in terms of the stage at which the two inputs could be summed in relation to the non-linearities defined by the hyperbolic ratio function. The authors performed two experiments, one aimed at testing how signals 
projecting at different spatial positions within the same eye are combined and a second aimed at testing how signals projecting at the same retinal location from both eyes are combined in the brain. The greatest predictive power (fit) was afforded by a 'late summation' model in which the input signals were summed at both the numerator and denominator only after their individual exponentiation.

The above examples demonstrate how one can gain insights that go beyond the mere existence and measurement of the degree of neural interactions. Indeed, by comparing the results of computational models to the empirical data obtained in the experiment, one can obtain evidence providing greater support for one plausible mechanism over another.

\section{MSPC measures}

The MSPC measure introduced by Yang (Yang et al., 2016) and discussed above (section 2.2) quantifies the degree to which the IMs are driven by the fundamental frequencies.

Gordon et al (Gordon et al., 2018) have expanded the use of this measure in several ways. First, they introduced the distinction between what they termed MSPCstim and MSPCres - two measures that may allow a distinction between interactions occurring at lower and higher levels of hierarchical processing (Figure 3B). Specifically, this was done in the context of the Hierarchical-FrequencyTagging paradigm in which two different modulation methods are simultaneously applied on the stimuli ((Gordon et al., 2017); A brief description of the method is provided in section 4.2.3). The difference between the MSPCstim and MSPCres measures lies in which phases are considered the 'driving' phases of the IM response. For MSPCstim, the fundamental frequency phases used in the MSPC calculation are those of the stimulus (i.e. the image on the screen) while MSPCres uses the phases of the EEG response (as obtained by the FFT) at the fundamental frequencies. The authors argue that the stimulus phases should be more tightly related to non-linear integration at earlier visual processing while the EEG response phases should reflect additional processing from the later stages. In other words, the MSPCstim and MSPCres measures may point at non-linear integration occurring at lower and at higher hierarchical levels, respectively. Indeed, the authors found that the MSPCstim and MSPCres measures of the IM response were differentially modulated by expectation and attention (Figure 3C), cognitive factors believed to impact descending and ascending signals, respectively (Feldman and Friston, 2010). More direct evidence for such a distinction may be afforded by additional analyses such as source localization and dynamic causal modelling (DCM).

Second, the authors performed further analyses to examine the sequence of computations leading to the 4th order IMs. Specifically, they tested which one of the following sequences are more likely: A) an early interaction between signals at the fundamental frequency followed by an additional nonlinearity, or B) an early non-linear process performed on each of the signals at the fundamental frequencies followed by an interaction between them. Then, they defined the driving frequencies as the second order IMs ( $f 2+f 1$ and $\mathrm{f} 2-\mathrm{f} 1$ ) for sequence $A$ and as the 2 nd order harmonics ( $2 \mathrm{f} 1$ and $2 \mathrm{f} 1$ ) for sequence $B$. They found that the MSPCres measure was significantly higher for sequence $B$ than $A$ and that the increase of MSPCres for 4th order IM due to allocation of endogenous attention measure was explained better by sequence $B$ than $A$. Consistent with the association of MSPCres with interactions occurring at higher hierarchical levels, these results support the option in which the signals driven by each of the stimulus modulation methods are first processed individually and then interact at a later stage. 
The above examples demonstrate how, despite IMs not pointing conclusively at specific neural processes to which they could be mapped, valuable insights can nevertheless be gained by applying additional controlled experiments, comparing empirical spectra against physiologically-plausible computational models, or complementing amplitude-based measures with MSPC analyses. These methods enable one to test different plausible neural integration mechanisms and to examine whether the observed experimental IM effects are more likely to be caused by one mechanism rather than another.

\section{IMs in perception research- from low to high-level processes}

IM measures have been used to examine various levels of perceptual processing. Here, we first present several representative studies exploring low- and mid-level visual processing. We then review more recent studies that examine the influence of cognitive factors on non-linear integration also at higher levels of the visual hierarchy.

\subsection{Low- and mid-level visual processing}

Most IM studies have focused on low and mid-level visual processes, including simple feedforward receptive field interactions (Regan and Regan, 1989), figure-ground segregation (Appelbaum et al., 2008), perceptual binding (Aissani et al., 2011; Boremanse et al., 2014) and integration of multiple visual features (Giani et al., 2012) (Figure 5).

A

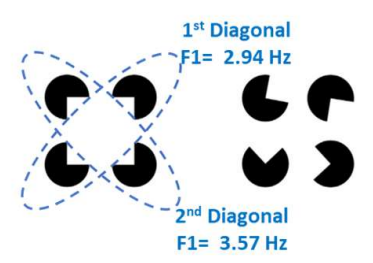

B

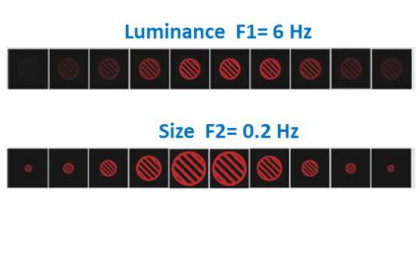

C

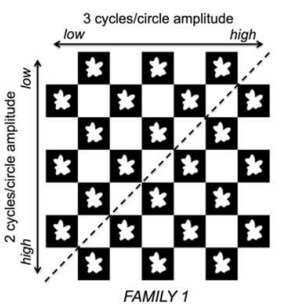

Left side $\mathrm{F} 2=7.5 \mathrm{~Hz}$

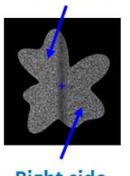

Right side $F 1=5.45 \mathrm{~Hz}$

Figure 5- IM experimental designs

A sample of various IM paradigms used in visual perception studies. (A) Gestalt formation. Alp et al. examined IMs as a neural signature of the global integration required for illusory surface perception using a variant of the Kanizsa figure and modulating the two diagonal 'pacman' shapes at different frequencies (reproduced with permission from (Alp et al., 2016). The blue text was added here for clarification.) (B) Multiple feature integration. Giani et al. used IMs to investigate the integration of multiple stimulus features within and across sensory modalities (reproduced with permission from (Giani et al., 2012). The blue text was added here for clarification.) The example above depicts the stimulus used to examine the integration of visual size and luminance. Each feature was modulated using distinct frequencies. (C) Perceptual learning. Vergeer et al. applied an IM analysis to examine how different spatial features are combined during holistic shape representation and perceptual learning (reproduced with permission from (Vergeer et al., 2018). The blue text was added here for clarification.) The table on the left shows a family of unfamiliar shapes that are highly similar to each other. Participants were trained to discriminate between two categories of these shapes (separated by the diagonal line in the table). Successful categorization relied on the combination of spatial features across both halves of the shape. Activity related to the processing of the two sides of each shape was tagged using different contrast-modulation frequencies (right figure). 
The IM studies carried out throughout the 1980s and 1990s focused primarily on low-level visual processing occurring at early visual areas (e.g. simple feedforward receptive field interactions). Early studies typically used visual stimuli involving gratings, windmill- and dartboard-like stimuli, in which luminance or contrast were modulated using two sine-wave frequencies while measuring visual evoked potentials in the EEG (Regan and Regan, 1989; Zemon and Ratliff, 1984). The significance of these studies was two-fold. First, they demonstrated how IMs provide a direct physiological measure of neural signal integration in the human brain. For example, several studies investigating early integration from both eyes demonstrated large differences in IM responses when the two eyes receive the same input (monoptic presentation), when only one eye receives input (monocular presentation) and when the two eyes receive different inputs (dichoptic presentation) (Suter et al., 1996) or, when comparing between healthy and stereoblind patients (Baitch and Levi, 1988).

Second, they demonstrated how plausible their models of neural processes can be by examining the response spectrum across frequencies (including harmonics and high order IMs, as detailed above in the section 3.3)(Regan and Regan, 1988a).

The utilization of IMs did not gain much traction in the years following these initial publications but a series of studies by Anthony Norcia and others, published from the late 1990s onward, further demonstrated the promising utility of IMs in vision research (Norcia et al., 2000; Norcia and Hou, 2002; Victor and Conte, 2000) .

\subsubsection{Figure-ground segregation}

Appelbaum et al. (Appelbaum et al., 2008) used simple texture-based images consisting of random luminance bars to investigate the basic mechanisms allowing object segmentation (Figure 5A). By tagging such "figure" and "ground" regions with different temporal frequencies $(3.6 \mathrm{~Hz}$ and $3 \mathrm{~Hz})$ and applying source-localization analyses (incorporating MRI-enabled individual head models), the authors could identify distinct neural networks responsible for the processing of figure and background regions, as well as figure-ground interactions. Specifically, the 2nd order IM term f1+f2 was found to be the most dominant interaction term, strongly depending on orientation cues within the stimuli's regions (figure or ground) as well as on gaps between the regions. This IM component was widely distributed throughout occipital areas.

Interestingly, the 4 th order term $2 f 1+2 f 2$ was present for all cue types, including when regions were separated solely based on the modulation frequencies (i.e. with no additional orientation cues). The $2 \mathrm{f} 1+2 \mathrm{f} 2$ term was also less affected by the figure and ground segmentation than the $f 1+f 2$ term. The authors interpreted the fact that these two IM components exhibited different functional specificities as evidence that at least two distinct figure-ground interaction mechanisms were involved. In accordance with several other studies (Hou et al., 2003; Victor and Conte, 2000), the authors found their data to be consistent with a cascade of two non-linear stages such as an initial within-region rectification followed by a second non-linear stage that pools neural activities across regions.

\subsubsection{Perceptual binding}

Several studies provide evidence linking IMs to perceptual binding processes that cannot be explained by the simple integration of common receptive-field signals (Aissani et al., 2011; Boremanse et al., 2014). For example, two studies demonstrated that specific IM components are modulated by the formation of illusory images- a process that cannot be attributed to bottom-up 
processes alone. Gundlach and Muller presented two 'pacman'-like shapes that faced each other, forming an illusory rectangle (Gundlach and Müller, 2013). In an important control condition, a circular 'ring' was added in between the pacman shapes, impeding the formation of the illusory rectangle. The two pacman shapes were then flickered at two different frequencies (square-wave luminance modulation at $8.5 \mathrm{~Hz}$ and $14.17 \mathrm{~Hz}$ ) and an increase in the $\mathrm{f} 1+\mathrm{f} 2$ and $2 \mathrm{f} 1+2 \mathrm{f} 2 \mathrm{IM}$ components was observed when the formation of the illusory rectangle was made easy. Importantly, this increase could not be explained by an increase at the fundamental tagging frequencies, emphasising the role of integration processes.

In a more recent study, Alp et al. used Kanizsa figures (Kanizsa, 1955) while contrast-modulating the two pairs of diagonal pacman-like shapes using different frequencies (2.94 and $3.57 \mathrm{~Hz}$ ) ((Alp et al., 2016); Figure 5B). By rotating these shapes around themselves, the formation of the illusory rectangle was made possible only during defined time periods (at which neighbouring straight edges were aligned). Previous studies have suggested that such perception of an illusory surface manifests at multiple visual areas and likely involves feedback loops between higher-level and lower-level visual areas (Lee \& Mumford, 2003; Stanley \& Rubin, 2003). Significant responses in this study were found at a range of second and third-order IM components ( $f 2-f 1, f 1+f 2,2 f 2-f 1,2 f 1+f 2$ and $f 1+2 f 2$ ). Interestingly, the average IM response increased when the illusory rectangle could be formed (the statistical significance of the effect was greatest for the $\mathrm{f} 1+\mathrm{f} 2$ and $2 \mathrm{f} 1+\mathrm{f} 2$ components). The authors conclude that some of the long-range signal interactions only take place when the distant visual elements of the stimulus form a coherent perception.

It is worth noting that the responses at the fundamental frequencies were also somewhat higher in this study when the illusory surface was perceivable, making it more difficult to attribute the increase in the IM responses to interactions that are unique to the perception of the illusory surface. To address this issue, the authors examined the relation between the differences in the fundamental and the differences in the IM responses across participants. Given no such relation was found, the authors concluded that the increase in the IM responses in the illusory condition is not a mere consequence of changes in the representation of the individual stimulus elements.

\subsubsection{Integration of multiple sensory features}

IMs generated by the interaction of two different visual features have been demonstrated in several studies. For example, Giani et al investigated the integration of multiple sensory features within and across sensory modalities (Giani et al., 2012) and found strong intermodulation components when simultaneously modulating the luminance and the size of a visual stimulus (Figure 6C) or when simultaneously modulating the frequency and the amplitude of an auditory stimulus.

In addition, in a brain-computer-interface study by Chen et al. (Chen et al., 2013), eight gratings were simultaneously presented on the screen and modulated using both an on/off flicker (either 10 $\mathrm{Hz}, 12 \mathrm{~Hz}$ or $15 \mathrm{~Hz}$ ) and a colour modulation (red and green colours alternating at a pace of either $0.5 \mathrm{~Hz}$ or $1 \mathrm{~Hz}$ ). By assigning different luminance and colour frequency combinations to each grating (including conditions with no colour modulation), the authors were able to achieve a high average classification accuracy of $93.8 \%$ based on the amplitude SNR of the IM frequencies at the $\mathrm{Oz}$ and 5 surrounding electrodes. This study demonstrates how IMs generated by the integration of multiple visual features can also be harnessed for applied research. 


\subsubsection{Perceptual learning}

In a recent attempt to utilise IMs for the study of perceptual learning, Vergeer et al. created highly similar shapes (based on a Fourier Boundary Descriptor method) and trained participants to distinguish between sets of stimuli ((Vergeer et al., 2018); Figure 5D). Importantly, the ability to correctly categorise and discriminate between such shapes is believed to require holistic representations rather than the simple detection of local features and requires participants to rely on combinations of spatial features for optimal performance. Only after training and perceptual learning were participants able to form unique holistic shape representations for each set of stimuli. The right and left parts of the images were contrast-modulated at different frequencies (5.45 and $7.5 \mathrm{~Hz}$ ). The average amplitude SNR of various high-order IMs ( $4^{\text {th }}-8^{\text {th }}$ order) were higher when participants observed stimuli belonging to a family of shapes they have learned to categorise (regardless of whether they have been trained on those specific exemplars or not). The authors suggest that this reflects high-level visual interactions related to holistic shape categorization. As a cautionary note, the authors did however acknowledge that the effects involved low-power IM components that were analysed in a post-hoc manner. Nevertheless, this study demonstrates the potential of IMs to be generated by relatively high-level visual processes.

The studies presented in this section demonstrate the application of IM technique to perception research from early studies of low-level visual processing to studies of mid-level processes such as perceptual binding and feature integration. In the following section we will see how IMs have been used in more recent years to investigate also higher-level processes.

\subsection{Awareness, attention and expectation}

Several recent studies demonstrate the utility of IM components for studying processes involving higher visual areas as well as factors such as awareness, expectation and attention (by 'attention' in this section we refer primarily to volitional, task-relevant, top-down attention as opposed to automatic, exogenously captured, bottom-up attention). We present a range of studies using binocular rivalry paradigms, or experimental manipulations of spatial attention, feature/objectbased attention, and expectation.

Notably, the sensory inputs to the visual system in most of these studies were kept constant across conditions. Hence, the observed changes in the IMs can be linked specifically to the changes in awareness, expectation, and attention rather than to changes in the physical input reaching the sensory system. Indeed, the topographical distribution of the IM components in most of these studies spanned areas beyond occipital regions, reaching parietal, central and frontal areas as well.

\subsubsection{Visual awareness during binocular rivalry}

Binocular rivalry is a type of perceptual rivalry in which perception alternates between different images presented to each eye and is often used to study processes related to unconscious and conscious perception. Several studies have investigated IMs in binocular rivalry (Katyal et al., 2016; Sutoyo and Srinivasan, 2009; Zhang et al., 2011). These reports reveal intriguing neural interactions that occur during rivalry. For example, Sutoyo and Srinivasan presented four half-circle gratings- one to each eye/hemifield combination, each of which differed according to its colour (either red or green) and orientation (one of two orthogonal diagonal orientations) ((Sutoyo and Srinivasan, 2009); Figure 7A). These combinations allowed percepts to be formed either by the combination of two 
hemifields within the same eye or by the combination of two complementary hemifields- one from each eye. Each grating was tagged using a different frequency (sinusoidal luminance modulations at $7.99 \mathrm{~Hz}, 9.22 \mathrm{~Hz}, 10.90 \mathrm{~Hz}$, and $11.99 \mathrm{~Hz}$ ), allowing distinct $\mathrm{f} 1+\mathrm{f} 2$ combinations to be identified for each eye/hemifield combination.

Intriguingly, the IM power at the $\mathrm{f} 1+\mathrm{f} 2 \mathrm{IM}$ component was modulated not by changes in conscious perception per se but rather by the type of rivalry taking place. In other words, the IMs differed between periods of eye-based rivalry (i.e., perception alternating between the stimuli shown to each of the two eye) and periods of pattern-based rivalry (i.e., perception alternating between the complementary hemifield combinations across both eyes). The spatial topography of the $f 1+f 2 I M$ component was notably more widespread than many of the results obtained in studies of low-level visual processes, and spanned also parietal and central electrodes. (It is not clear if the f2-f1 or higher order IM terms were analysed in this study.) Such distributions are more similar to those found in other studies of high-level visual perception, supporting the notion that the IMs in this study reflect neural processes occurring at higher hierarchical levels. The authors conclude that both eye-based rivalry and pattern-based rivalry involve competition between percepts and that the neural processes related to the binding of the visual hemifields into the two rival percepts take place during binocular rivalry even when only one percept is consciously perceived.

It is interesting to compare the results from this study and those of the binocular rivalry study by Zhang et al described above in section 3.3. At first glance, the results of these studies may seem inconsistent with each other as the IMs reported in Sutoyo et al are linked to the existence of rivalry while the IMs reported in Zhang et al are linked to the cessation of rivalry. A more in-depth comparison, on the other hand, highlights why any question such as 'what do the IMs signify' must always be considered in respect to the individual study at question.

In this case, the studies by Zhang et al. and Sutoyo et al. differed in a range of important parameters, including the modulation methods applied (e.g., two stimuli presented one to each eye versus four stimuli presented two to each eye, respectively; square contrast-reversal modulation at 6.6 and 7.5 $\mathrm{Hz}$ versus sinusoidal luminance modulation at $8-12 \mathrm{~Hz}$ ), the behavioral procedure (tasks involving attended and unattended conditions versus only attended conditions), the IM components analyzed (a range of low and higher-order IMs versus only the $\mathrm{f} 1+\mathrm{f} 2$ component), and the channels used for the analysis (only 6 electrodes near Oz versus all electrodes). While the channels used for the analysis in Zhang et al are known to be sensitive to activity at early visual areas, the more widespread topography suggested by Sutoyo et al suggests integration processes involving higher levels as well.

In Sutoyo et al., the stimuli appearing at the complementary hemifields were always congruent in either one or both visual features (orientation and color), and the observed IMs were only those that could arise from the integration of complementary hemifields (either within or across eyes). Conversely, Zhang et al. presented only one stimulus to each eye, each with a different form and color, and one modulation frequency was used for each eye's stimulus (hence the IMs could not distinguish between hemifields).

Therefore, the results of both Zhang et al. and Sutoyo et al. indicate that signal integration, as reflect by the IMs, depends on the type of rivalry taking place. In Zhang et al, no IMs could be formed from the stimulus presented to a single eye. Therefore, if the IMs in these studies are linked to the rivalry 
taking place then the IMs in Zhang et al. can be expected to be reduced during eye rivalry. Only when attention was removed, the integration of the incongruent stimuli from both eyes increased, most likely at low visual areas.

A possible interpretation of these results is that the interactions reflected by the IMs in both studies may relate less to the actual conscious perception per se. but rather to the potential percepts (consistent with the Bayesian approach to rivalry; (Hohwy et al., 2008)). The mechanisms underlying the observed IMs, and the hierarchical levels at which the 'potentiality' of specific percepts are determined most likely differ across these studies. While yet highly speculative, this can be tested by analyzing dynamic changes in IMs across the different hierarchical levels.

\subsubsection{Spatial attention}

Consistent with the notion that attention modulates the propagation of signal processing along the cortical hierarchy, a recent study by Kim et al (Kim et al., 2017) has demonstrated an attentiondependant emergence of IMs in parietal and prefrontal regions. In this electrocorticography (ECOG) study, the authors used wedge-shaped gratings, placed adjacent to each other but flickering at different frequencies, to assess the role of selective spatial-attention on stimulus integration (Figure 6B). A remarkable dissociation between the propagation of the first harmonics and of the f2f1 IM term from the visual cortex to parietal and prefrontal areas was demonstrated.

When comparing conditions in which participants were prompted to attend away from the wedges to conditions in which they were prompted to attend either to one or to both wedges, the authors found that selective attention strongly enhanced the signals in the parietal and prefrontal regions but not in the occipital region. Specifically, when participants were instructed to attend to one of the wedges, the strongest attention-specific enhancement was observed for the first harmonic (of the flicker at the attended region). When attending to both wedges, the strongest attention-specific enhancement was observed for the f1-f2 IM component.

Notably, this IM response was evident in the parietal and prefrontal regions even without firstharmonic responses (which would typically reflect responses to individual stimuli). The authors note that the presence of the IM response in the absence of the first harmonic responses in prefrontal and parietal cortex implies that SSVEP components are propagated from other brain regions to prefrontal and parietal cortex, where the first harmonic components are gated out. While such an explanation is plausible, another possibility might be that this reflects a mechanism in which the two inputs are processed primarily with respect to one another (i.e. with a significant interaction term but with no significant 'main effect' terms that reflect separate processing of the individual inputs). As demonstrated in Figure 2 (rows 6 and 8), certain systems (or certain types of modulation) can indeed yield an output containing primarily IMs.

A control condition in which fully overlapping gratings were presented (using two flicker frequencies) suggested that the IM response in the prefrontal cortex reflects not only a stimulusdriven integration of two adjacent stimuli but rather the additional attention-dependent response to two behaviourally relevant stimuli. Consistent with previous studies (Desimone and Duncan, 1995; Maunsell and Cook, 2002), the authors suggest these results reflect the hierarchical processes in which the neural representation of the visual scene becomes more highly task-specific as one progresses up the cortical hierarchy. The prefrontal and parietal regions, under this framework, 
combine stimulus-specific responses according to what is currently relevant for their behavioural goal, representing either individual stimuli or their interaction.

Interestingly, a similar distinction between responses at the fundamental frequencies within the occipital lobe and responses at specific IMs within the parietal or the frontal lobes was evident in a MEG study by Aissani et al. (Aissani et al., 2011). There, the authors presented participants with a series of bars arranged in a square shape. The horizontal and vertical bar pairs moved and oscillated vertically and horizontally at two different frequencies and participants reported whether the bars were perceived as a single moving shape ('bound') or not ('unbound'). While the power at the fundamental motion frequencies was strongest in the occipital lobe and did not differ between bound and unbound perception, the $\mathrm{f} 1+\mathrm{f} 2 \mathrm{IM}$ component was localized at the precentral sulcus region and was stronger during perception of bound motion compared to unbound motion.

\subsubsection{Feature/object-based attention and expectation}

The Hierarchical Frequency Tagging (HFT) method has recently been utilized to explore the mechanisms by which expectation and attention modulate the integration of top-down and bottomup signals in perception. The core idea of the HFT method is the combination of two frequency tagging methods - one for tagging activity occurring primarily at low visual areas, and another for tagging activity occurring at higher visual areas. In Gordon et al. (Gordon et al., 2017; Gordon et al., 2018) the authors used SSVEP contrast modulations at 10-15 Hz for the former, and Semantic Wavelet-Induced Frequency Tagging (SWIFT; (Koenig-Robert and VanRullen, 2013)) modulations at 0.8-1.3 $\mathrm{Hz}$ for the latter. (A video demonstration can be found here:

https://figshare.com/s/3c217f2a379dd6735b29.)

In a series of HFT experiments performed by Gordon et.al (Gordon et al., 2018) participants were presented various images of houses and faces and were required to perform different behavioral tasks through which the authors manipulated expectation (the predictability of upcoming images) and attention (the counted image category) (Figure 3C). Importantly, the cognitive manipulations did not involve any changes to the visual input itself. Using the MSPC analysis to quantify the degree to which the phases of the IMs are driven by the phases of the fundamental frequencies (as described in section 3.3) the authors showed that expectation and attention increased the MSPCstim and the MSPCres measures, respectively. Consistent with the predictive coding framework of perception, where attention and expectation are believed to modulate ascending and descending signals, respectively (Feldman and Friston, 2010; Schroger et al., 2015), the authors suggest that the MSPCstim and MSPCres measures reflect signal integration occurring at lower and at higher levels of the cortical hierarchy, respectively.

In summary, the studies described in section 4 demonstrate how IMs can aid the investigation of various aspects of expectation, awareness and attention (task-relevance) including, but no limited to, binocular rivalry, spatial attention and feature/object-based attention.

We further suggest that these IM studies provide unique support to the notion of attention as a dynamic mechanism that constrains the vast amount of possibilities for signal integration, allowing only relevant stimulus combinations to propagate up the cortical hierarchy, and defining the types of perceptual inferences that engage in competition. 
A
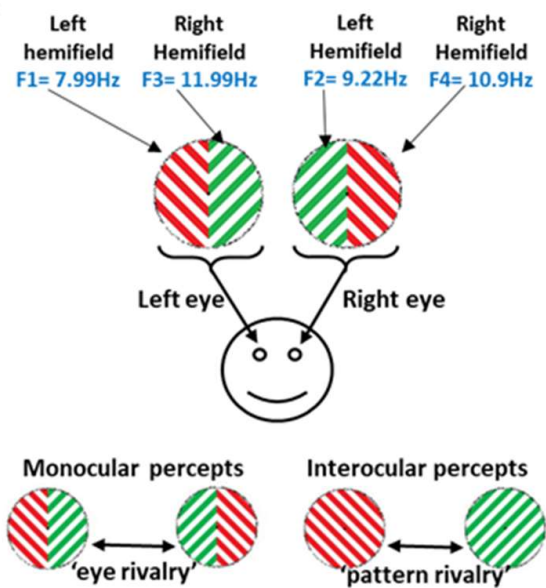

Interocular percepts
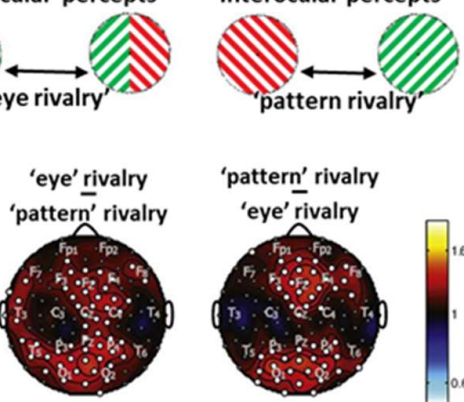

'eye' IMs

(e.g. f1+f3)

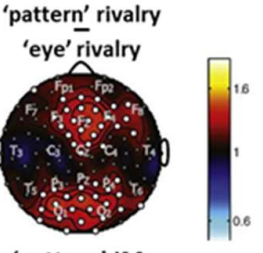

'pattern' IMs

(e.g. f1+f4)
B
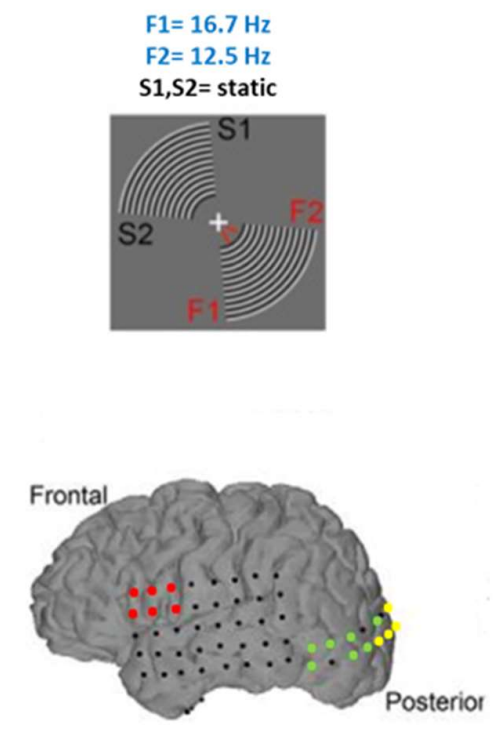

significant (f2-f1) IM only

significant $f 1$ or $f 2$ fundamental only significant IM and either fundamental

Figure 6

(A) Binocular rivalry. Sutoyo and Srinivasan presented different gratings to each hemifield and eye combination and modulated each grating using a different frequency (stimulus and results reproduced with permission from (Sutoyo and Srinivasan, 2009). Text was added, and the figure was reorganised here for clarity) This way, distinct f1+f2 IM components indicated the integration of signals induced by specific grating combinations. The top and middle rows depict one of 16 different color and orientation configurations used in the study. IMs increased as a function of the type of rivalry that was taking place (i.e. eye- vs pattern- based rivalry; bottom row) rather than the specific conscious perception. (B) Spatial attention. Kim et al tested the effect of spatial attention on the integration of adjacent visual stimuli (reproduced with permission from (Kim et al., 2017). The figure was reorganised here for clarity). The f2-f1 IM component was found to be the frequency component most enhanced when attending to both wedges. This enhancement was evident in the parietal and prefrontal regions even in the lack of first-harmonic responses. The authors suggest that these results imply that the neural representation of the visual scene becomes more task-specific as one progresses up the cortical hierarchy.

\section{Limitations, outstanding questions, and future directions}

We have highlighted the unique ability of IMs to provide a direct and objective physiological measure for neural interactions and we have demonstrated the ability to utilize IMs for the study of low and mid-level perceptual processes, as well as high-level processes and the involvement of cognitive factors such as expectation and attention. We have highlighted the need to consider the IMs within each study in conjunction with the fundamental and harmonic frequencies, and we have outlined the methodologies that have been used to shed light on the possible mechanisms giving rise to the IMs. In Table 2 we list a range of potential future projects that are important for advancing the use of IMs in neuroscience even further.

These methods do not come without limitations. First, a general limitation is posed by the measuring tool itself. For example, the majority of IM studies presented above rely on EEG measurements. 
These, in turn, reflect only a small portion of neural activity- that which is synchronised across a substantial number (>100K) of spatially aligned cortical neurons (Nunez and Srinivasan, 2006). Interactions across multiple signals are bound to occur also in much smaller scales than this, and in neural populations that may not contribute to a measurable M/EEG signal (e.g. subcortical neurons). For example, Giani et al. (Giani et al., 2012) simultaneously presented and modulated two visual features and two auditory features using different sets of modulation frequencies (two for each modality). The IMs they observed reflected within-modality but not between-modality interactions. This null result does not necessarily mean the visual and auditory signals did not interact at any cortical level but rather that interactions possibly elicited by that paradigm were not at a scale detectable by the measuring tool used in that study (MEG).

Another aspect worth considering is the predominant use of the Fourier transform for extracting frequency-domain amplitude and phase in the spectrum from the raw time-series. While the Fourier transform is an extremely useful tool for this purpose, the ability of additional analyses, which do not assume sinusoidal rhythms, to provide a complementary characterization of the neural oscillations has been raised in relation to various endogenous oscillatory waveforms (Cole and Voytek, 2017). This may be true for IM studies as well since even if sinusoidal stimulus modulations are used, there is no a priori reason to assume that all entrained neural responses will be perfectly sinusoidal as well, particularly when focusing on high-level cortical processes and using advanced tagging methods such as the semantic wavelet-induced frequency tagging (Koenig-Robert and VanRullen, 2013).

Future research may provide valuable answers to various other open questions. For example, can information theoretic and causal measures, such as transfer entropy (Schreiber, 2000), be applied to infer the causality among the various harmonic and IM components? Is there a limit to the spatial scales from which IMs in neural data can be used to inform perception research? We've presented MEG, EEG and ECoG studies in this review. Can IMs be reliably obtained, for example, also from local field potentials (LFP) on the one hand and from $\mathrm{FMRI}$ on the other? What is the relation between IMs and other measures of integration such as integrated information? As integrated information is suggested as a possible measure of consciousness (Tononi, 2012), can IMs also serve as a measure of consciousness? Spectral decomposition of integrated information is currently being developed and is a promising approach for this direction (Cohen, Sasai, Tsuchiya, Oizumi - in preparation)

The above questions should be tackled at various levels, ranging from theoretical and mathematical through computational simulations and modelling to experimental and empirical data, coupled with advanced analysis methods. IMs have been proven to be a valuable means for identifying and characterising interactions between distinct neural signals in perceptual and cognitive processing, and because they are a direct measure of these interactions, they hold promise for unravelling the complex hierarchical structure involved in various brain processes. 
Table 2. Open questions and Potential future IM projects

\section{Systematic studies of fundamentals, harmonics, and IMs.}

Given the multitude of experimental methodologies currently applied in the field, any attempt to interpret IMs can only be made in respect to individual studies after carefully considering the specific set of parameters used in that study. Is it possible to reach a more general prescription for answering a question such as 'what do the IMs signify'?

Exploring this possibility requires developing efficient experimental and analyses methods and creating a platform that will enable more systematic comparisons across studies.

Various existing methods can be modified and expanded for use in IM studies. This includes ideas such as retinotopic mapping, frequency sweeping, phase-amplitude cross-frequency coupling, and others. Establishing a multi-site, pre-registered, large study, which incorporates replication of seemingly inconsistent studies (e.g., Sutoyo et al. (Sutoyo and Srinivasan, 2009) versus Zhang et al. (Zhang et al., 2011)) with the same subjects, parameters, stimuli, and analysis techniques may provide a functional account of IMs that can be generalized better across future studies.

2. Neuronal circuit level understanding of the origin of the fundamentals, harmonics, and IMs, and how they appear at macro M/EEG and ECOG scales.

The need to better understand how neuronal circuit-level activity translates into macro-level signals has been raised in the general context of EEG (Cohen, 2017). Much research has focused on understanding the origins of EEG features such the different frequency-band oscillations (alpha, gamma, etc.). What can IMs tell us about their unique underlying circuit-level computations?

3. Inter-individual differences in fundamentals, harmonics, and IMs and their sensitivity to different paradigms and parameters.

Can the individual difference in IMs be used as a potential marker for individual traits related to binding, attention, expectation, and learning? For example, can IMs explain different learning rates in tasks that require forming new associations between sensory features within and across modalities?

4. Relationship between ongoing oscillations (alpha, beta, etc) and evoked fundamentals, harmonics, and IMs.

Endogenous oscillations have been shown to influence SSVEP studies. Does this apply in the same manner to IMs? Is it indeed the case that IM studies should avoid using alpha range frequency modulations, for example?

\section{Acknowledgments}

This study was supported by funding from Australian Research Council FT120100619, DP180100396, DP180104128 Naotsugu Tsuchiya, DP160102770 Jakob Hohwy, and Templeton World Charity Foundation, Inc (TWCF0199) Naotsugu Tsuchiya. 


\section{References}

Aissani, C., Cottereau, B., Dumas, G., Paradis, A.L., Lorenceau, J., 2011. Magnetoencephalographic signatures of visual form and motion binding. Brain Res 1408, 27-40.

Alp, N., Kogo, N., Van Belle, G., Wagemans, J., Rossion, B., 2016. Frequency tagging yields an objective neural signature of Gestalt formation. Brain Cogn 104, 15-24.

Appelbaum, L.G., Wade, A.R., Pettet, M.W., Vildavski, V.Y., Norcia, A.M., 2008. Figure-ground interaction in the human visual cortex. Journal of Vision 8, 8.1-819.

Baitch, L.W., Levi, D.M., 1988. Evidence for nonlinear binocular interactions in human visual cortex. Vision Res 28, 1139-1143.

Baker, D.H., Wade, A.R., 2017. Evidence for an Optimal Algorithm Underlying Signal Combination in Human Visual Cortex. Cerebral Cortex 27, 254-264.

Boremanse, A., Norcia, A.M., Rossion, B., 2013. An objective signature for visual binding of face parts in the human brain. Journal of Vision 13, 6-6.

Boremanse, A., Norcia, A.M., Rossion, B., 2014. Dissociation of part-based and integrated neural responses to faces by means of electroencephalographic frequency tagging. Eur J Neurosci 40, 29872997.

Boynton, G.M., Engel, S.A., Glover, G.H., Heeger, D.J., 1996. Linear systems analysis of functional magnetic resonance imaging in human V1. J Neurosci 16, 4207-4221.

Calvert, G.A., Hansen, P.C., Iversen, S.D., Brammer, M.J., 2001. Detection of Audio-Visual Integration Sites in Humans by Application of Electrophysiological Criteria to the BOLD Effect. Neuroimage 14, 427-438.

Campbell, D., Crutchfield, J., Farmer, D., Jen, E., 1985. Experimental mathematics: The role of computation in nonlinear science. Communications of the Association for Computing Machinery 28, 374-384.

Candy, T.R., Skoczenski, A.M., Norcia, A.M., 2001. Normalization Models Applied to Orientation Masking in the Human Infant. The Journal of Neuroscience 21, 4530-4541.

Canolty, R.T., Knight, R.T., 2010. The functional role of cross-frequency coupling. Trends Cogn Sci 14, 506-515.

Carandini, M., Heeger, D.J., 2011. Normalization as a canonical neural computation. Nat Rev Neurosci 13, 51-62.

Chen, X., Chen, Z., Gao, S., Gao, X., 2013. Brain-computer interface based on intermodulation frequency. J Neural Eng 10, 066009. 
Cohen, M.X., 2017. Where Does EEG Come From and What Does It Mean? Trends Neurosci 40, 208218.

Cole, S.R., Voytek, B., 2017. Brain Oscillations and the Importance of Waveform Shape. Trends Cogn Sci 21, 137-149.

Cunningham, D.G.M., Baker, D.H., Peirce, J.W., 2017. Measuring nonlinear signal combination using EEG. J Vis 17, 10.

Desimone, R., Duncan, J., 1995. Neural mechanisms of selective visual attention. Annu Rev Neurosci $18,193-222$.

Ding, J., Sperling, G., Srinivasan, R., 2006. Attentional modulation of SSVEP power depends on the network tagged by the flicker frequency. Cereb Cortex 16, 1016-1029.

Feldman, H., Friston, K.J., 2010. Attention, Uncertainty, and Free-Energy. Frontiers in Human Neuroscience 4, 215.

Fuchs, S., Andersen, S.K., Gruber, T., Muller, M.M., 2008. Attentional bias of competitive interactions in neuronal networks of early visual processing in the human brain. Neuroimage 41, 1086-1101.

Gabbiani, F., Krapp, H.G., Hatsopoulos, N., Mo, C.H., Koch, C., Laurent, G., 2004. Multiplication and stimulus invariance in a looming-sensitive neuron. J Physiol Paris 98, 19-34.

Giani, A.S., Ortiz, E., Belardinelli, P., Kleiner, M., Preissl, H., Noppeney, U., 2012. Steady-state responses in MEG demonstrate information integration within but not across the auditory and visual senses. Neuroimage 60, 1478-1489.

Gordon, N., Koenig-Robert, R., Tsuchiya, N., van Boxtel, J.J., Hohwy, J., 2017. Neural markers of predictive coding under perceptual uncertainty revealed with Hierarchical Frequency Tagging. eLife 6.

Gordon, N., Tsuchiya, N., Koenig-Robert, R., Hohwy, J., 2018. Expectation and attention increase the integration of top-down and bottom-up signals in perception through different pathways. bioRxiv.

Gulbinaite, R., van Viegen, T., Wieling, M., Cohen, M.X., VanRullen, R., 2017. Individual Alpha Peak Frequency Predicts $10 \mathrm{~Hz}$ Flicker Effects on Selective Attention. J Neurosci 37, 10173-10184.

Gundlach, C., Müller, M.M., 2013. Perception of illusory contours forms intermodulation responses of steady state visual evoked potentials as a neural signature of spatial integration. Biol Psychol 94, 55-60.

Herrmann, C.S., 2001. Human EEG responses to 1-100 Hz flicker: resonance phenomena in visual cortex and their potential correlation to cognitive phenomena. Exp Brain Res 137, 346-353.

Hohwy, J., Roepstorff, A., Friston, K., 2008. Predictive coding explains binocular rivalry: an epistemological review. Cognition 108, 687-701. 
Hou, C., Pettet, M.W., Sampath, V., Candy, T.R., Norcia, A.M., 2003. Development of the spatial organization and dynamics of lateral interactions in the human visual system. J Neurosci 23, 86308640.

James, T.W., Stevenson, R.A., 2012. The Use of fMRI to Assess Multisensory Integration. In: Murray, M.M., Wallace, M.T. (Eds.), The Neural Bases of Multisensory Processes. Boca Raton (FL): CRC Press/Taylor \& Francis;.

Kanizsa, G., 1955. Margini quasi-percettivi in campi con stimolazione omogenea. Rivista di Psicologia 49, 7-30.

Katyal, S., Engel, S.A., He, B., He, S., 2016. Neurons that detect interocular conflict during binocular rivalry revealed with EEG. Journal of Vision 16, 18-18.

Katyal, S., Vergeer, M., He, S., He, B., Engel, S.A., 2018. Conflict-sensitive neurons gate interocular suppression in human visual cortex. Sci Rep 8, 1239.

Kim, Y.J., Tsai, J.J., Ojemann, J., Verghese, P., 2017. Attention to Multiple Objects Facilitates Their Integration in Prefrontal and Parietal Cortex. J Neurosci 37, 4942-4953.

Koenig-Robert, R., VanRullen, R., 2013. SWIFT: a novel method to track the neural correlates of recognition. Neuroimage 81, 273-282.

Kouh, M., Poggio, T., 2008. A Canonical Neural Circuit for Cortical Nonlinear Operations. Neural Computation 20, 1427-1451.

Lea-Carnall, C.A., Montemurro, M.A., Trujillo-Barreto, N.J., Parkes, L.M., El-Deredy, W., 2016. Cortical Resonance Frequencies Emerge from Network Size and Connectivity. PLOS Computational Biology 12, e1004740.

Markram, H., 2003. Elementary Principles of Nonlinear Synaptic Transmission. In: Hecht-Nielsen, R., McKenna, T. (Eds.), Computational Models for Neuroscience: Human Cortical Information Processing. Springer London, London, pp. 125-169.

Maunsell, J.H., Cook, E.P., 2002. The role of attention in visual processing. Philos Trans R Soc Lond B Biol Sci 357, 1063-1072.

Mitra, P.P., Pesaran, B., 1999. Analysis of dynamic brain imaging data. Biophys J 76, 691-708.

Muthukumaraswamy, S.D., 2013. High-frequency brain activity and muscle artifacts in MEG/EEG: a review and recommendations. Front Hum Neurosci 7, 138.

Noppeney, U., 2012. Characterization of Multisensory Integration with fMRI. In: Murray, M.M., Wallace, M.T. (Eds.), The Neural Bases of Multisensory Processes. Boca Raton (FL): CRC Press/Taylor \& Francis.

Norcia, A.M., Appelbaum, L.G., Ales, J.M., Cottereau, B.R., Rossion, B., 2015. The steady-state visual evoked potential in vision research: A review. Journal of Vision 15, 4. 
Norcia, A.M., Harrad, R.A., Brown, R.J., 2000. Changes in cortical activity during suppression in stereoblindness. Neuroreport 11, 1007-1012.

Norcia, A.M., Hou, C., 2002. Non-linear analysis of the contrast paradox for vernier acuity. Journal of Vision 2.

Nunez, P.L., Srinivasan, R., 2006. Electric fields of the brain : the neurophysics of EEG, 2nd ed. Oxford University Press, Oxford ; New York.

Palva, S., Palva, J.M., 2012. Discovering oscillatory interaction networks with M/EEG: challenges and breakthroughs. Trends Cogn Sci 16, 219-230.

Pena, J.L., Konishi, M., 2004. Robustness of multiplicative processes in auditory spatial tuning. J Neurosci 24, 8907-8910.

Regan, D., Regan, M.P., 1988a. Objective evidence for phase-independent spatial frequency analysis in the human visual pathway. Vision Research 28, 187-191.

Regan, M.P., Regan, D., 1988b. A frequency domain technique for characterizing nonlinearities in biological systems. Journal of Theoretical Biology 133, 293-317.

Regan, M.P., Regan, D., 1989. Objective investigation of visual function using a nondestructive zoomFFT technique for evoked potential analysis. Can J Neurol Sci 16, 168-179.

Schreiber, T., 2000. Measuring information transfer. Phys Rev Lett 85, 461-464.

Schroger, E., Marzecova, A., SanMiguel, I., 2015. Attention and prediction in human audition: a lesson from cognitive psychophysiology. Eur J Neurosci 41, 641-664.

Shapley, R., 2009. Linear and nonlinear systems analysis of the visual system: why does it seem so linear? A review dedicated to the memory of Henk Spekreijse. Vision Res 49, 907-921.

Srinivasan, R., Bibi, F.A., Nunez, P.L., 2006. Steady-state visual evoked potentials: distributed local sources and wave-like dynamics are sensitive to flicker frequency. Brain Topography 18, 167-187.

Suter, S., Suter, P.S., Perrier, D.T., Parker, K.L., Fox, J.A., Roessler, J.S., 1996. Differentiation of VEP intermodulation and second harmonic components by dichoptic, monocular, and binocular stimulation. Vis Neurosci 13, 1157-1166.

Sutoyo, D., Srinivasan, R., 2009. Nonlinear SSVEP responses are sensitive to the perceptual binding of visual hemifields during conventional 'eye' rivalry and interocular 'percept' rivalry. Brain research $1251,245-255$.

Tononi, G., 2012. Integrated information theory of consciousness: an updated account. Arch Ital Biol 150, 56-90.

Tsai, J.J., Wade, A.R., Norcia, A.M., 2012. Dynamics of normalization underlying masking in human visual cortex. The Journal of Neuroscience 32, 2783-2789. 
Vergeer, M., Kogo, N., Nikolaev, A.R., Alp, N., Loozen, V., Schraepen, B., Wagemans, J., 2018. EEG frequency tagging reveals higher order intermodulation components as neural markers of learned holistic shape representations. Vision Res.

Vialatte, F.-B., Maurice, M., Dauwels, J., Cichocki, A., 2010. Steady-state visually evoked potentials: Focus on essential paradigms and future perspectives. Progress in Neurobiology 90, 418-438.

Victor, J.D., Conte, M.M., 2000. Two-frequency analysis of interactions elicited by Vernier stimuli. Vis Neurosci 17, 959-973.

Yang, Y., Solis-Escalante, T., Yao, J., Daffertshofer, A., Schouten, A.C., van der Helm, F.C.T., 2016. A General Approach for Quantifying Nonlinear Connectivity in the Nervous System Based on Phase Coupling. International Journal of Neural Systems 26, 1550031.

Zemon, V., Ratliff, F., 1982. Visual evoked potentials: evidence for lateral interactions. Proc Natl Acad Sci U S A 79, 5723-5726.

Zemon, V., Ratliff, F., 1984. Intermodulation components of the visual evoked potential: responses to lateral and superimposed stimuli. Biol Cybern 50, 401-408.

Zhang, P., Jamison, K., Engel, S., He, B., He, S., 2011. Binocular rivalry requires visual attention. Neuron 71, 362-369.

Zhu, M., Rozell, C.J., 2013. Visual nonclassical receptive field effects emerge from sparse coding in a dynamical system. PLoS Comput Biol 9, e1003191. 